\title{
Information Transmission in Coalitional Voting Games*
}

\author{
Roberto Serrano ${ }^{\dagger}$ and Rajiv Vohra ${ }^{\ddagger}$ \\ Department of Economics \\ Brown University \\ Providence, RI 02912, U.S.A. \\ Working Paper No. 2005-01
}

January 2005

\begin{abstract}
A core allocation of a complete information economy can be characterized as one that would not be unanimously rejected in favor of another feasible alternative by any coalition. We use this test of coalitional voting in an incomplete information environment to formalize a notion of resilience. Since information transmission is implicit in the Bayesian equilibria of such voting games, this approach makes it possible to derive core concepts in which the transmission of information among members of a coalition is endogenous. Our results lend support to the credible core of Dutta and Vohra (2003) and the core proposed by Myerson (2003) as two that can be justified in terms of coalitional voting.

JEL Classification: C71, C72, D51, D82.

Keywords: Core, Incomplete Information, Coalitional Voting, Resilience, Mediation.

${ }^{*}$ We acknowledge support from NSF grant SES-0133113. Serrano also thanks the Institute for Advanced Study for its hospitality and Deutsche Bank for research support.

$\dagger$ Department of Economics, Brown University, roberto_serrano@brown.edu; www . econ. brown. edu/faculty/serrano

$\ddagger$ Department of Economics, Brown University, rajiv_vohra@brown.edu; www . econ. brown. edu/ ${ }^{\sim}$ rvohra
\end{abstract}




\section{Introduction}

In the complete information setting, the core provides a natural way of formalizing coalitional stability. Simple as the core concept is, there is no unique, unambiguous way of extending it to an exchange economy with incomplete information at the interim stage; see Forges, Minelli and Vohra (2002) for a survey. This paper is concerned with the issue of making endogenous the amount of information that agents share in the process of cooperation. We shall formalize coalitional stability by means of equilibria of voting games in which agents choose between the status-quo and another feasible alternative.

At the interim stage, there are two sets of assumptions to be made (amount of information sharing, and whether or not incentive constraints are imposed) before the standard definition of the core can be extended to the incomplete information model. For example, we may assume that there is no sharing of information; agents in a coalition can only rely on common knowledge events to construct potential objections. This approach leads to the notion of the coarse core, first formalized by Wilson (1978) in a framework without incentive constraints and subsequently extended by Vohra (1999) to include them. The main idea underlying the coarse core, namely that a coalition's objection is focused on a common knowledge event, is motivated by standard issues of adverse selection; see the examples in Wilson (1978) and Vohra (1999).

Not surprisingly, the theory is quite different if one allows coalition members to share information. If information sharing is unrestricted among the members of a coalition, one arrives at the notion of the fine core, also proposed by Wilson (1978). Incentive constraints can be added to a fine objection by requiring that it be incentive compatible over the event that is relevant for the objection. While unrestricted sharing of information may seem arbitrary and, in many instances, unreasonable, there do exist cases in which some amount of information sharing seems natural.

Our aim is to make endogenous the amount of information that is shared among agents when a coalition forms in order to block a status-quo allocation. ${ }^{1}$ While this concern is by no means new (see, for example, Dutta and Vohra (2003)), our approach here is based on viewing the core in another,

\footnotetext{
${ }^{1}$ Our approach is related to the notions of durability in Holmström and Myerson (1983) and credibility in Dutta and Vohra (2003), as we shall discuss below.
} 
more positive (perhaps more primitive) way. We argue that non-cooperative equilibrium theory is ideally suited to deal with the question of how much private information agents transmit to each other. This is so even though we are interested in modeling cooperative behavior. ${ }^{2}$ This leads to the idea of formalizing coalitional stability by making use of non-cooperative equilibrium behavior in a voting game.

Suppose a status-quo allocation is in the core, and a coalition compares it to some other feasible allocation. Clearly, if the two alternatives were to be voted upon by the agents in the coalition, we would not expect a unanimous acceptance of the alternative over the status-quo. Indeed, in the complete information setting this is a defining property of the core. We take this simple test of stability and apply it systematically to a model with incomplete information. The amount of information sharing as well as the incentive constraints will then emerge as equilibrium conditions of a voting game.

One might think that precise details of the voting game may turn out to be critical, perhaps leading to a plethora of different notions of core stability. Fortunately, we are able to show that this is not the case; the coalitional voting approach is quite sharp in its conclusions, and there are only a few important aspects of the voting game that matter for core stability, leading to a few possible core concepts. Indeed, a version of the revelation principle is at work, as a Bayesian equilibrium of an arbitrary voting mechanism can be replaced by its outcome-equivalent truthful equilibrium in a direct voting game, where the message simply consists of a type report and a "yes-no" vote to the alternative. Thus, while information transmission is endogenous in the Bayesian equilibrium actions of non-cooperative voting games, it is also robust to different specifications of their details.

If incentive constraints are not important (because types become verifiable) we show that the coalitional voting approach yields the fine core of Wilson (1978) as the natural concept. In the general case where types are not verifiable, resilience to coalitional voting yields the credible core of Dutta and Vohra (2003). In this sense, the credible core can be seen as the appropriate generalization of the fine core when incentive constraints are relevant (as will be explained, the credible core is not simply the incentive compatible

\footnotetext{
${ }^{2}$ While the ability to cooperate makes it reasonable to allow for unfettered, frictionless communication, it does not mean that agents will necessarily share their private information with others or believe others' claims that cannot be verified.
} 
fine core described above).

An interesting modification of this simple voting game allows a mediator to construct a more sophisticated challenge to a status-quo by implementing the alternative as a function of the agents' reported types. Effectively, the only difference between this game and the simpler one corresponding to the credible core is that in this case a coalition can challenge a status-quo over an informational event that is not necessarily a product event. We call the corresponding set of resilient allocations the mediated core. The importance of the product/nonproduct structure of the event over which an objection takes place justifies the following analogy: a mediated objection is to a credible objection as a correlated equilibrium is to a Nash equilibrium.

Finally, we allow a mediator to randomize over the coalitions that it approaches for a vote. This leads to a form of resilience that yields the randomized mediated core, a notion that corresponds closely with a recent core concept suggested by Myerson (2003). Myerson's approach is based on virtual utility, and he is able to prove non-emptiness of his core concept in his class of games, provided that severance payments take place in an additional commodity, a nummeraire, and feasibility is only required in expected terms. It follows from our results that his non-emptiness result also implies nonemptiness of the core concepts found here under his assumptions, since the blocking restrictions that define the mediated core and the credible core are less stringent than those in Myerson's. On the other hand, we also know that the non-emptiness question in general is difficult, given the results in Forges, Mertens and Vohra (2002) and Vohra (1999) showing that the incentive compatible coarse core, a superset of all these other concepts, may be empty.

\section{Preliminaries}

The basic model of an exchange economy with asymmetric information can be formulated as follows. Let $T_{i}$ denote the (finite) set of agent $i$ 's types. The interpretation is that $t_{i} \in T_{i}$ denotes the private information possessed by agent $i$. With $N=\{1, \ldots, n\}$ as the finite set of agents, let $T=\prod_{i \in N} T_{i}$ denote the set of all information states. We will use the notation $t_{-i}$ to denote $\left(t_{j}\right)_{j \neq i}$. Similarly $T_{-i}=\prod_{j \neq i} T_{j}, T_{S}=\prod_{j \in S} T_{j}$ and $T_{-S}=\prod_{j \notin S} T_{j}$. We assume that agents have a common prior probability distribution $q$ defined on $T$, and that no type is redundant, i.e., $q\left(t_{i}\right)>0$ for all $t_{i} \in T_{i}$ for all $i$. 
At the interim stage, nature chooses $t \in T$, and each agent $i$ knows her type, $t_{i}$. Hence, conditional probabilities will be important: for each $i \in N$ and $t_{i} \in T_{i}$, the conditional probability of $t_{-i} \in T_{-i}$, given $t_{i}$ is denoted $q\left(t_{-i} \mid t_{i}\right)$.

We assume that there are a finite number of commodities, and that the consumption set of agent $i$ is $X_{i}=R_{+}^{l}$. Agent $i$ 's utility function in state $t$ is denoted $u_{i}(\cdot, t): X_{i} \times T \mapsto R$. We shall assume that $u_{i}(x, t) \geq u_{i}(0, t)$ for all $i$, all $t \in T$ and all $x \in R_{+}^{L}$. The endowment of agent $i$ of type $t_{i}$ is $\omega_{i}$ (assumed to be independent of the state - with this assumption, all private information concerns agents' preferences.)

We can now define an exchange economy as $\mathcal{E}=\left\langle\left(u_{i}, X_{i}, \omega_{i}, T_{i}\right)_{i \in N}, q\right\rangle$.

For coalition $S \subseteq N$, a feasible (state contingent) $S$-allocation, $x: T \mapsto$ $R^{l s}$ (where $s$ denotes the cardinality of $S$ ), consists of a commodity bundle for each consumer in $S$ in each state such that $\sum_{i \in S} x_{i}(t) \leq \sum_{i \in S} \omega_{i}$ for all $t \in T$, and satisfying that $x\left(t_{S}, t_{-S}^{\prime}\right)=x\left(t_{S}, t_{-S}^{\prime \prime}\right)$ for all $t_{S} \in T_{S}$ and for all $t_{-S}^{\prime}, t_{-S}^{\prime \prime} \in$ $T_{-S}$. (The latter assumption is made to exclude basic externalities across coalitions, i.e., the set of feasible allocations to a coalition is independent of the information held by the complement). We will denote by $\mathcal{A}_{S}$ the set of feasible state contingent allocations of $S$. We shall use $A_{S}$ to denote the set of feasible allocations in a given state: $A_{S}=\left\{\left(x_{i}\right) \in R^{l s} \mid \sum_{i} x_{i} \leq \sum_{i} \omega_{i}\right\}$. Similarly, state contingent $N$-allocations are simply referred to as allocations, and the set of state contingent allocations is denoted by $\mathcal{A}$.

Given $x \in \mathcal{A}_{S}$, the interim utility of agent $i \in S$ of type $t_{i}$ is:

$$
U_{i}\left(x \mid t_{i}\right)=\sum_{t_{-i} \in T_{-i}} q\left(t_{-i} \mid t_{i}\right) u_{i}\left(x_{i}\left(t_{-i}, t_{i}\right),\left(t_{-i}, t_{i}\right)\right) .
$$

If agent $i$ of type $t_{i}$ pretends to be of type $t_{i}^{\prime}$ (while all other agents are truthful), she gets interim utility:

$$
U_{i}\left(x, t_{i}^{\prime} \mid t_{i}\right)=\sum_{t_{-i} \in T_{-i}} q\left(t_{-i} \mid t_{i}\right) u_{i}\left(x_{i}\left(t_{-i}, t_{i}^{\prime}\right),\left(t_{-i}, t_{i}\right)\right) .
$$

An $S$-allocation $x \in A_{S}$ is incentive compatible if for every $i \in S$ and for every $t_{i}, t_{i}^{\prime} \in T_{i}$ :

$$
U_{i}\left(x \mid t_{i}\right) \geq U_{i}\left(x, t_{i}^{\prime} \mid t_{i}\right)
$$

We shall denote the set of incentive compatible $S$-allocations by $\mathcal{A}_{S}^{*}$, and the set of incentive compatible allocations by $\mathcal{A}^{*}$.

For an event $E \subseteq T$ and $t_{i} \in T_{i}$, let

$$
E_{-i}\left(t_{i}\right)=\left\{t_{-i} \in T_{-i} \mid\left(t_{i}, t_{-i}\right) \in E\right\}
$$


and

$$
E_{i}=\left\{t_{i} \in T_{i} \mid E_{-i}\left(t_{i}\right) \neq \emptyset\right\} .
$$

Consider an allocation rule $x \in \mathcal{A}$, agent $i$ of type $t_{i}$ and an event $E$. Suppose $q\left(E_{-i}\left(t_{i}\right)\right)>0$. Then the interim utility conditional on $E$ can be expressed as:

$$
U_{i}\left(x \mid t_{i}, E\right)=\sum_{t_{-i} \in E_{-i}\left(t_{i}\right)} q\left(t_{-i} \mid t_{i}\right) u_{i}\left(x_{i}\left(t_{-i}, t_{i}\right),\left(t_{-i}, t_{i}\right)\right) .
$$

(Strictly speaking, the expression on the right hand side should be divided by $q\left(E_{-i}\left(t_{i}\right) \mid t_{i}\right)$, but we will find it convenient not to do so.)

The corresponding interim utility (conditional on $E$ ) if type $t_{i}$ pretends to be of type $t_{i}^{\prime}$ is:

$$
U_{i}\left(x, t_{i}^{\prime} \mid t_{i}, E\right)=\sum_{t_{-i} \in E_{-i}\left(t_{i}\right)} q\left(t_{-i} \mid t_{i}\right) u_{i}\left(x_{i}\left(t_{-i}, t_{i}^{\prime}\right),\left(t_{-i}, t_{i}\right)\right) .
$$

Given $E \subseteq T$, an $S$-allocation $x \in \mathcal{A}_{S}$ is incentive compatible over $E$ if for every $i \in S$ and for every $t_{i}, t_{i}^{\prime} \in E_{i}$ :

$$
U_{i}\left(x \mid t_{i}, E\right) \geq U_{i}\left(x, t_{i}^{\prime} \mid t_{i}, E\right) .
$$

The set of such allocations is denoted $\mathcal{A}_{S}^{*}(E)$.

We will often consider an event that refers to independent subsets of $T_{i}$, and is therefore a product event of the form $E=\prod_{i} E_{i}$, where $E_{i} \subseteq T_{i}$ for all $i$.

\section{Coalitional Voting}

One way to assess the coalitional stability of a status-quo is to ask whether or not some coalition would vote unanimously in favor of another feasible 'outcome'. This is a somewhat pedantic exercise in the complete information framework because there it is obvious that the core is precisely the set of allocations which no coalition would vote (unanimously) to give up in favor of some other feasible allocation. Moreover, this conclusion does not depend on precisely how the coalitional voting game is constructed. Our aim is to take this simple characterization of core stability in the complete information case and extend it to a model with incomplete information. ${ }^{3}$ Since several

\footnotetext{
${ }^{3}$ The test being performed here is weaker than the implementation exercise. In the classical framework it is possible to construct a game form that implements the core of an
} 
new issues arise in this transition, it is useful to begin by illustrating the coalitional voting approach in the simple setting where types are verifiable. The main focus of the paper, however, is the more general case in which types are not verifiable.

\subsection{Verifiable Types}

We take verifiability of types to mean that eventually all private information becomes public, and contracts contingent on types can be enforced (by using prohibitive penalties if necessary). In this setting agents cannot misrepresent their types, and incentive constraints become unnecessary. Despite this simplicity, it is by no means obvious how the core should be defined in such a model. Indeed, Wilson (1978) suggested two distinct notions of the core: the fine core (allowing arbitrary information sharing) and the coarse core (allowing agents to coordinate only on common knowledge events); see Forges et al (2002) for precise definitions based on the types formulation. Wilson's notion of the core, and others that have since been suggested, are all based on the traditional approach based on dominance and feasibility of a potential objection. The main conceptual issue in the construction of an objection concerns an exogenously specified way of information transmission within the coalition (see also Lee and Volij (2002) and Volij (2000)). ${ }^{4}$ The coalitional voting approach is different in that it seeks to justify the notion of an objection from more primitive principles: an equilibrium in a voting game is used as a means for defining what an 'objection' is. As we shall see, in the verifiable types case, this approach leads to just one corresponding notion of the core - Wilson's fine core. We see this as an illustration of the power of the coalitional voting approach in selecting an appropriate notion of core stability.

Suppose $x \in \mathcal{A}$ is the status-quo and coalition $S$ can consider making use of a voting game to discard $x$ in favor of $y \in \mathcal{A}_{S}$. More precisely, members

economy; see, for example, Perry and Reny (1994), and Serrano and Vohra (1997). The present paper may be seen as a preliminary step in developing game forms to implement the 'core' in incomplete information economies.

${ }^{4}$ An alternative route is taken by de Clippel (2004), who proposes a competitive screening procedure with outside brokers that absorb bankruptcies in objections. This allows him to transform each agent's decision problem of whether joining an objection into a one-person decision problem, independent of more complex considerations, such as the ones tackled here in terms of information transmission. 
of $S$ vote to either "accept" or "reject" the new proposal $y$. It is important that $y$ is not seen as a proposal by a particular agent, in order to avoid complications of signaling that such an act would imply. ${ }^{5}$ The coalition is simply asked to make a choice between $x$ and $y$. In case of a unanimous "accept" vote, the outcome assigned will be $y(t)$ to coalition $S$, where $t$ is the profile of actual types. Note that type announcements are not necessary because we are assuming that the types eventually become publicly known. ${ }^{6}$ In case of a "reject" vote from any member of $S$ the mediator assigns $x(t)$ to the grand coalition. This defines a game $\Gamma_{x}^{0}(S, y)$, in which the only active players are all the players in $S$, who simultaneously must choose from $\{a, r\}$, ("accept" or "reject" the alternative $y$ ). Then, $y$ is implemented if and only if it is unanimously accepted; otherwise, $x$ is the outcome. A strategy for player $i$ is a function $v_{i}: T_{i} \mapsto\{a, r\}$. Given a profile of strategies $\left(v_{i}().\right)$, the outcome, $\phi(v)$, for members of $S$, is defined as follows:

$$
\phi_{i}(v(t))= \begin{cases}y_{i}(t) & \text { if } v_{i}\left(t_{i}\right)=a \text { for all } i \in S \\ x_{i}(t) & \text { otherwise }\end{cases}
$$

A strategy profile $\bar{v}$ of the voting game $\Gamma_{x}^{0}(S, y)$ is a Bayesian Nash equilibrium if, for all $i \in S$ and $t_{i} \in T_{i}$,

$$
U_{i}\left(\phi_{i}\left(\bar{v} \mid t_{i}\right)\right) \geq U_{i}\left(\phi_{i}\left(\bar{v}_{-i}, v_{i}\right) \mid t_{i}\right), \text { for all } v_{i}: T_{i} \mapsto\{a, r\}
$$

Given a Bayesian Nash equilibrium $\bar{v}$, define for each $i$ the set of types who vote to accept the alternative, i.e.,

$$
E_{i}(\bar{v})=\left\{t_{i} \in T_{i} \mid v_{i}\left(t_{i}\right)=a\right\}
$$

For $i \in S$, let $E_{-i}=\prod_{j \in S, j \neq i} E_{j} \times T_{-S}$.

In a voting game $\Gamma_{x}^{0}(S, y)$, a Bayesian Nash equilibrium $\bar{v}$ is said to be an equilibrium rejection of $x$ if there is positive probability that in equilibrium all agents vote to accept the alternative mechanism, i.e., $q\left(\prod_{i \in S} E_{i}(\bar{v})\right)>0$,

\footnotetext{
${ }^{5}$ An extension to coalitional settings of the type of analysis in Maskin and Tirole (1992) would be an important next step. In their approach, the informed principal proposes the agent a contract that, therefore, may signal some of his private information.

${ }^{6} \mathrm{~A}$ model in which agents have to announce their types in order to implement the allocation, but types become known at a later stage (and prohibitive penalties are available), is operationally identical to this because it can be assumed that agents announce their types truthfully.
} 
and $\phi(\bar{v})$ is not interim equivalent to $x$ for all $i \in S$ (in the sense that there exists $i \in S$ and $t_{i} \in T_{i}$ such that $\left.U_{i}\left(\phi_{i}(\bar{v}) \mid t_{i}\right)>U_{i}\left(x \mid t_{i}\right)\right)$ ).

A status-quo $x$ is resilient to coalitional voting with verifiable types if there does not exist a voting game $\Gamma_{x}^{0}(S, y)$ with an equilibrium rejection of $x$.

Proposition 1 Given $x \in \mathcal{A}$, a coalition $S$ and $y \in \mathcal{A}_{S}$, the following statements are equivalent:

(a) There is an equilibrium rejection of $x$ in the voting game $\Gamma_{x}^{0}(S, y)$.

(b) There exists $y^{\prime} \in \mathcal{A}_{S}$ and $E_{i} \subseteq T_{i}$ for all $i \in S$, where $q(E)>0$ for the product event $E=\prod_{i \in S} E_{i} \times T_{-S}$, such that:

$$
U_{i}\left(y^{\prime} \mid t_{i}, E\right) \geq U_{i}\left(x \mid t_{i}, E\right) \text { for all } i \in S \text { and all } t_{i} \in E_{i}
$$

with strict inequality for some $i$ and $t_{i}$.

Corollary $1 x$ is resilient to coalitional voting with verifiable types if and only if it belongs to the fine core.

The corollary follows from the fact that condition (b) is essentially the same as the definition of a fine objection; see, for example, Forges et al (2002). The only difference is that an 'objection' is usually defined with a strict inequality for all $i$. It should be clear that the characterization of equilibria of voting games will be in the form of weak inequalities. For this reason, throughout this paper, when we refer to a certain core concept it should be understood to be the 'strong' version of that core concept. ${ }^{7}$

It also worth remarking that statement (a) in the Proposition simply refers to an equilibrium of the voting game, and unlike statement (b) or the definition of a fine objection, it is not couched in terms of a particular event over which an 'objection' takes place. This is also a feature of our other equivalence results below.

Proof of Proposition 1: An equilibrium $\bar{\sigma}$ of the voting game $\Gamma_{x}^{0}(S, y)$ can be characterized equivalently in terms of $y$ and $x$ as follows. For all $i \in S$ and $t_{i} \in E_{i}$ :

$$
\sum_{t_{-i} \in E_{-i}} q\left(t_{-i} \mid t_{i}\right) u_{i}\left(y \mid t_{i}\right)+\sum_{t_{-i} \notin E_{-i}} q\left(t_{-i} \mid t_{i}\right) u_{i}\left(x \mid t_{i}\right) \geq \sum_{t_{-i} \in T_{-i}} q\left(t_{-i} \mid t_{i}\right) u_{i}\left(x \mid t_{i}\right),
$$

\footnotetext{
${ }^{7}$ Monotonicity of preferences will suffice to eliminate this difference as well, in the present result. However, when we turn to the case of non-verifiable types, this difference may be important.
} 
and for all $i \in S, t_{i} \notin E_{i}$ :

$$
\sum_{t_{-i} \in E_{-i}} q\left(t_{-i} \mid t_{i}\right) u_{i}\left(y \mid t_{i}\right)+\sum_{t_{-i} \notin E_{-i}} q\left(t_{-i} \mid t_{i}\right) u_{i}\left(x \mid t_{i}\right) \leq \sum_{t_{-i} \in T_{-i}} q\left(t_{-i} \mid t_{i}\right) u_{i}\left(x \mid t_{i}\right) .
$$

These two conditions are equivalent to the following two conditions:

$$
U_{i}\left(y \mid t_{i}, E\right) \geq U_{i}\left(x \mid t_{i}, E\right) \text { for all } i \in S \text { and } t_{i} \in E_{i}
$$

and

$$
U_{i}\left(y \mid t_{i}, E\right) \leq U_{i}\left(x \mid t_{i}, E\right) \text { for all } i \in S \text { and } t_{i} \notin E_{i} .
$$

Clearly, this means that (a) implies (b).

To see that (b) implies (a) consider $y^{\prime}$ and $E$ satisfying condition (b), and define:

$$
y(t)= \begin{cases}y^{\prime}(t) & \text { if } t \in E \\ 0 & \text { otherwise. }\end{cases}
$$

Since $U_{i}\left(y^{\prime} \mid t_{i}, E\right)=U_{i}\left(y \mid t_{i}, E\right)$ for all $i \in S$ and $t_{i} \in E_{i}$, it follows that $y$ satisfies both (1) and (2), and this completes the proof.

\subsection{Non-Verifiable Types}

When types are not verifiable, we need to be more careful about precisely what a status-quo means. Henceforth, we shall take a status-quo, $x$, to refer to an incentive compatible, state contingent allocation (thus $x \in \mathcal{A}^{*}$ ) with the interpretation that in every state $t$, the outcome is $x(t)$, unless there is an agreement to change it. This means, in particular, that if there is an attempt to change the status-quo but the attempt fails, the outcome in state $t$ is $x(t)$, i.e., any discussion about a possible change does not by itself allow any agent to strategically manipulate the status-quo $x .^{8}$ Since $x$ was assumed to be incentive compatible, this can be justified by a scenario in which agents have already sent their truthful reports to implement the status-quo via a direct mechanism. And this cannot be changed if the status-quo prevails. This assumption greatly simplifies the analysis, and will be used throughout the rest of this paper.

\footnotetext{
${ }^{8}$ Cramton and Palfrey (1995) consider the opposite case: if an alternative is accepted no manipulation is possible but a rejection yields information that can be used in manipulating the 'status-quo'.)
} 
As before, coalitional voting may involve a comparison between a statusquo (a feasible allocation rule for the grand coalition) and an allocation rule that is feasible (both informationally and physically) for the given coalition. However, when types are not verifiable, an allocation rule is only the simplest example of what a coalition may consider as an alternative. More generally, we may think of a coalition constructing a communication mechanism of its own to map out its feasible set of alternatives. Indeed, this will capture the idea that a coalition is permitted as much latitude in constructing a competing proposal as is within the bounds of feasibility. A voting game can now be used to describe the situation in which members of a coalition vote to reject a status-quo in favor of a competing mechanism. The new mechanism is applied if all agents vote for it; otherwise the status-quo remains as the outcome. We will say that a status-quo is resilient to coalitional voting if there does not exist a coalition and a Bayesian Nash equilibrium of a voting game in which an alternative mechanism is unanimously accepted in some positive probability state. Note that our approach yields a very strong notion of stability; a resilient allocation rule cannot be rejected in any Bayesian Nash equilibrium of any competing mechanism in any (positive probability) state. We now turn to formal definitions.

A mechanism for coalition $S$ consists of message sets $M_{i}$ for each $i \in S$ and an outcome function $g: M \mapsto A_{S}$.

A status-quo $x \in \mathcal{A}^{*}$, and a mechanism for $S,\left(\left(M_{i}\right)_{i \in S}, g\right)$, define a voting game in which each player $i \in S$ chooses an action in $\{a, r\} \times M_{i}$. The interpretation being that $a$ refers to an 'accept' vote in favor of the new mechanism while $r$ refers to a rejection of the new proposal. A strategy for player $i$ is therefore $\sigma_{i}: T_{i} \mapsto\{a, r\} \times M_{i}$. We will find it convenient to denote $\sigma_{i}\left(t_{i}\right)=\left(v_{i}\left(t_{i}\right), m_{i}\left(t_{i}\right)\right)$, where the first element denotes the vote of agent $i$ of type $t_{i}$. Given a strategy profile $\sigma$, let $\phi(\sigma)$ denote the corresponding outcome for coalition $S$. In state $t$, the commodity bundle assigned to each $i \in S$ is denoted $\phi_{i}(\sigma(t))$, and defined as follows: ${ }^{9}$

$$
\phi_{i}(\sigma(t))= \begin{cases}g_{i}(m(t)) & \text { for all } t \text { such that } v_{i}\left(t_{i}\right)=a \text { for all } i \in S \\ x_{i}(t) & \text { otherwise }\end{cases}
$$

A coalitional voting game for coalition $S$ corresponding to status-quo, $x$, and a mechanism $\left(\left(M_{i}\right), g\right)$ can now be denoted $\Gamma_{x}\left(S,\left(M_{i}\right)_{i \in S}, g\right)$. This is

\footnotetext{
${ }^{9}$ Note that $\phi(\sigma(t))$ does not necessarily belong to $A_{S}$ since $\left(x_{i}(t)\right)_{i \in S}$ need not belong to $A_{S}$.
} 
the formulation first used by Holmström and Myerson (1983), for the grand coalition, in defining their notion of durability. And as they pointed out, this way of formulating a voting game is more general than it may first seem. It includes any complex voting procedure for various alternatives, as long as each agent has the option, at the outset, to anonymously veto the new proposal and revert to the status-quo. The mechanism can then be interpreted as the normal form of the game following the accept/reject vote.

A Bayesian Nash equilibrium of the voting game $\Gamma_{x}$ is a strategy profile, $\sigma$, for members of $S$ such that, for all $i \in S$ and $t_{i} \in T_{i}$,

$$
U_{i}\left(\phi_{i}(\sigma) \mid t_{i}\right) \geq U_{i}\left(\phi_{i}\left(\sigma_{-i}, \sigma_{i}^{\prime}\right) \mid t_{i}\right), \text { for all } \sigma_{i}^{\prime}: T_{i} \mapsto M_{i} .
$$

In a direct mechanism $\left(\left(T_{i}\right), y\right)$, a truthful equilibrium is an equilibrium where $m_{i}\left(t_{i}\right)=t_{i}$ for all $i \in S$ and all $t_{i} \in T_{i}$.

Given a Bayesian Nash equilibrium $\sigma=\left(\left(v_{i}\left(t_{i}\right), m_{i}\left(t_{i}\right)\right)\right.$, define for each $i$ the set of types who vote $a$, i.e.,

$$
E_{i}(\sigma)=\left\{t_{i} \in T_{i} \mid v_{i}\left(t_{i}\right)=a\right\} .
$$

Let $E(\sigma)=\prod_{i \in S} E_{i}(\sigma) \times T_{-S}$ denote the states in which the alternative is adopted.

In a voting game $\Gamma_{x}\left(S,\left(M_{i}\right)_{i \in S}, g\right)$, a Bayesian Nash equilibrium $\sigma$ is said to be an equilibrium rejection of $x$ if there is positive probability that in equilibrium all agents vote to accept the alternative mechanism, i.e., $q(E(\sigma))>0$, and $\phi(\sigma)$ is not interim equivalent to $x$ for all $i \in S$.

A status-quo $x$ is resilient to coalitional voting if there does not exist a voting game, $\Gamma_{x}\left(S,\left(M_{i}\right)_{i \in S}, g\right)$, with an equilibrium rejection of $x$.

Proposition 2 Given $x \in \mathcal{A}^{*}$, and a coalition, $S$, the following statements are equivalent:

(a) There is an equilibrium rejection of $x$ in voting game $\Gamma_{x}\left(S,\left(M_{i}\right)_{i \in S}, g\right)$.

(b) There is a truthful equilibrium rejection of $x$ in a (direct) voting game $\Gamma_{x}\left(S,\left(T_{i}\right)_{i \in S}, y\right)$.

(c) There exists $y^{\prime} \in \mathcal{A}_{S}$ and $E_{i} \subseteq T_{i}$ for all $i \in S$, where $q(E)>0$ for the product event $E=\prod_{i \in S} E_{i} \times T_{-S}$, such that:

(i) $U_{i}\left(y^{\prime} \mid t_{i}, E\right) \geq U_{i}\left(x \mid t_{i}, E\right)$ for all $i \in S$ and all $t_{i} \in E_{i}$, with strict inequality for some $i$ and $t_{i}$. 
(ii) $U_{i}\left(y^{\prime} \mid t_{i}, E\right) \leq U_{i}\left(x \mid t_{i}, E\right)$ for all $i \in S$ and all $t_{i} \notin E_{i}$.

(iii) $y^{\prime} \in \mathcal{A}_{S}^{*}(E)$.

It is instructive to compare this proposition to the revelation principle. The equivalence between (a) and (b) follows from the usual argument, and allows us to restrict attention to direct mechanisms, without loss of generality. It shows that a wide variety of coalitional voting games are strategically equivalent to one in which agents vote and report their types. In terms of Bayesian Nash equilibria, it is unimportant whether the vote and type reports are simultaneous or sequential (votes followed by type reports or vice versa). In equilibrium, acceptance of an alternative mechanism is equivalent to truthful acceptance in a direct voting game.

The equivalence of these with (c) has a more novel interpretation: it can be viewed as a form of a revelation principle concerning mechanisms in the context of a status-quo. The existence of an equilibrium rejection (through some mechanism) of a status-quo is equivalent to the existence of a feasible allocation rule satisfying the three inequalities in condition (c): (i) there is an event over which all members of the coalition gain, (ii) it is reasonable for them to believe this event since those types who do not belong to this event would not vote to accept the alternative mechanism, and (iii) the new proposed mechanism is incentive compatible over the event $E$.

Dutta and Vohra (2003), define $x \in \mathcal{A}^{*}$ to be in the credible core if there does not exist a coalition $S, y^{\prime} \in \mathcal{A}_{S}$ and an event $E=\prod_{i \in S} E_{i} \times T_{-S}$ (where $q(E)>0$ and $E_{i} \subseteq T_{i}$ for all $i \in S$ ) such that

(i) $\frac{U_{i}\left(y^{\prime} \mid t_{i}, E\right)}{q\left(E_{-i}\left(t_{i}\right) \mid t_{i}\right)}>\frac{U_{i}\left(x_{i} \mid t_{i}, E\right)}{q\left(E_{-i}\left(t_{i}\right) \mid t_{i}\right)}$ for all $i \in S$ and all $t_{i} \in E_{i}$.

(ii) $\frac{U_{i}\left(y^{\prime} \mid t_{i}, E\right)}{q\left(E_{-i}\left(t_{i}\right) \mid t_{i}\right)} \leq \frac{U_{i}\left(x_{i} \mid t_{i}, E\right)}{q\left(E_{-i}\left(t_{i}\right) \mid t_{i}\right)}$ for all $i \in S$ and $t_{i} \notin E_{i}$ such that $E_{-i}\left(t_{i}\right) \neq \emptyset$.

(iii) $y^{\prime} \in \mathcal{A}_{S}^{*}(E)$.

Note that if agent $i$ is of type $t_{i} \notin E_{i}$ and $E_{-i}\left(t_{i}\right)=\emptyset$, then her vote has bearing on her outcome. Thus condition (ii) above is the same as condition c (ii) of Proposition 2. This observation yields the following corollary.

Corollary $2 x$ is resilient to coalitional voting if and only if it belongs to the credible core. 
Proof of Proposition 2. Suppose $\sigma=\left(v_{i}\left(t_{i}\right), m_{i}\left(t_{i}\right)\right)$ is an equilibrium of $\Gamma_{x}\left(S,\left(M_{i}\right)_{i \in S}, g\right)$. Let $E_{i}=\left\{t_{i} \in T_{i} \mid v_{i}\left(t_{i}\right)=a\right\}$, and let $y(t)=g(m(t))$ for all $t$. The equilibrium interim utility of agent $i$ of type $t_{i}$ is:

$$
U_{i}\left(\sigma \mid t_{i}\right)= \begin{cases}U_{i}\left(y \mid t_{i}, E\right)+U_{i}\left(x \mid t_{i}, T \backslash E\right) & \text { if } t_{i} \in E_{i} \\ U_{i}\left(x \mid t_{i}\right) & \text { otherwise. }\end{cases}
$$

The fact that $\sigma$ is an equilibrium means:

(1) An agent $i \in S$ of type $t_{i} \in E_{i}$ cannot gain by rejecting the alternative,

$$
U_{i}\left(y \mid t_{i}, E\right) \geq U_{i}\left(x \mid t_{i}, E\right) \text { for all } i \in S \text { and all } t_{i} \in E_{i},
$$

or by continuing to accept but changing the choice of $m_{i}\left(t_{i}\right)$ to $m_{i}\left(t_{i}^{\prime}\right)$,

$$
U_{i}\left(y \mid t_{i}, E\right) \geq U_{i}\left(y, t_{i}^{\prime} \mid t_{i}, E\right) \text { for all } i \in S, t_{i} \in E_{i}, t_{i}^{\prime} \in T_{i} .
$$

(2) An agent $i$ of type $t_{i} \notin E_{i}$ cannot gain by accepting the alternative, and choosing $m_{i}\left(t_{i}^{\prime}\right)$,

$$
U_{i}\left(x \mid t_{i}, E\right) \geq U_{i}\left(y, t_{i}^{\prime} \mid t_{i}, E\right) \text { for all } i \in S, t_{i} \notin E_{i}, t_{i}^{\prime} \in T_{i} .
$$

Since $i$ of type $t_{i} \notin E_{i}$ cannot change the outcome by continuing to reject the alternative, this exhausts all possible unilateral deviations. Thus conditions (1), (2) and (3) characterize an equilibrium of the voting mechanism. If the equilibrium involves a rejection of the status-quo, then in addition to these three conditions we must have $q(E)>0$ and at least one inequality in (1) being strict. Clearly, then (a) implies (b) as well as (c). Of course, (b) implies (a), and so it remains only to be shown that (c) implies (b).

To see that (c) implies (b), consider $y^{\prime}$ and $E$ satisfying condition (c). Let

$$
y(t)= \begin{cases}y^{\prime}(t) & \text { if } t \in E \\ 0 & \text { otherwise. }\end{cases}
$$

Since $U_{i}\left(y^{\prime} \mid t_{i}, E\right)=U_{i}\left(y \mid t_{i}, E\right)$ for all $i \in S$ and $t_{i} \in E_{i}$, it follows from (i) of condition (c) that $y$ satisfies (1), with at least one strict inequality. From (ii) of condition (c) we know that $y^{\prime}$ (or $y$ ) satisfies (3) for all $t_{i}^{\prime} \in E_{i}$. The fact that $y$ also satisfies it for all $t_{i}^{\prime} \notin E_{i}$ follows from construction. Similarly, (2) follows from (iii) of condition (c).

We proceed to give an example that illustrates the difference between the credible core and the incentive compatible fine core. It is a simple version 
of a "lemon" asymmetric information economy, and it is taken from Vohra (1999).

Example 1: Let $N=\{1,2\}$. Agent 1 is fully informed and has two possible types: $T_{1}=\left\{t_{H}, t_{L}\right\}$. Agent 2 is uninformed about the true type of agent 1 and assigns equal probability to both. Let $t_{H}$ also denote the high state and $t_{L}$ the low state. Agent 1 is the seller of an indivisible good to be traded for money, and agent 2 is the buyer. In state $t_{H}$, agents' valuations for the indivisible good are $v_{1}$ and $v_{2}$, respectively, while they are 0 in state $t_{L}$. We shall assume that $v_{2} / 2<v_{1}<v_{2}$. Under these assumptions, the credible core consists exclusively of no-trade contracts in state $t_{H}$ and no transfers of money in state $t_{L}$. To see this, suppose there were trade in state $t_{H}$. Individual rationality for the seller implies that the money transfer should be at least $v_{1}$. Using incentive compatibility, one can establish that the same transfer must happen in state $t_{L}$, which makes it impossible for the buyer to meet his individual rationality constraint. Note how any fine objection (constructed in state $t_{H}$ ) would not be credible because type $t_{L}$ would like to join. However, as just observed, any credible core contract would not be in the incentive compatible fine core.

\section{Mediated Voting/Blocking}

There is one respect in which the voting mechanism of the previous section is not general enough. While agents are allowed a lot of flexibility in choosing a communication mechanism, the fact that the application of the alternative mechanism is based on independent accept/reject decisions may turn out to be an important restriction. One can argue that if a mediator can be used to translate messages into actions, presumably the mediator could also be delegated the accept/reject decision (perhaps as a function of additional inputs into the mechanism).

Recall that in the voting game $\Gamma_{x}\left(S,\left(M_{i}\right)_{i \in S}, g\right)$ of the previous section, the outcome function of the mechanism $(M, g)$ maps from $M$ to $A_{S}$. However, an outcome of the game includes that the possibility that the alternative is rejected, and the status-quo survives. A more general communication mechanism would allow the outcome function to map from $M$ to $A_{S} \cup\{x\} .{ }^{10}$ This is the interpretation of a mechanism we shall adopt in this section. As

\footnotetext{
${ }^{10}$ There is some abuse of notation here because rejecting the new mechanism leads to $x(t)$ in state $t$.
} 
before, an agent votes for or against a new mechanism and also chooses a message to communicate in the mechanism. Given a status-quo $x$, coalition $S$ and a mechanism $(M, g)$, where $g: \mapsto A_{S} \cup\{x\}$, we denote the coalitional voting game $\Gamma_{x}^{\prime}\left(S,\left(M_{i}\right)_{i \in S}, g\right)$.

Given a strategy profile $\sigma$, let $\phi(\sigma)$ denote the corresponding outcome for coalition $S$. Let $E(\sigma)$ denote the states in which the mechanism effectively adopts a new alternative, i.e.,

$$
E(\sigma)=\{t \in T \mid \phi(\sigma(t)) \neq x(t)\} .
$$

Note that $t \in E(\sigma)$ implies that $v_{i}\left(t_{i}\right)=\{a\}$ for all $i \in S$, but the converse may not hold, i.e., acceptance of an 'alternative' by all agents in $S$ does not necessarily mean that the mechanism accepts an alternative. Let

$$
E_{i}(\sigma)=\left\{t_{i} \in T_{i} \mid\left(t_{i}, t_{-i}\right) \in E \text { for some } t_{-i} \in T_{-i}\right\}
$$

In a voting game $\Gamma_{x}^{\prime}\left(S,\left(M_{i}\right)_{i \in S}, g\right)$, a Bayesian Nash equilibrium $\sigma$ is said to be an equilibrium rejection of $x$ if $q(E(\sigma))>0$ and $\phi(\sigma)$ is not interim equivalent to $x$ for all $i \in S$.

A status-quo $x$ is resilient to mediated coalitional voting if there does not exist a voting game, $\Gamma_{x}^{\prime}\left(S,\left(M_{i}\right)_{i \in S}, g\right)$, with an equilibrium rejection of $x$.

Proposition 3 Given $x \in \mathcal{A}^{*}$, and a coalition, $S$, the following statements are equivalent:

(a) There is an equilibrium rejection of $x$ in a mediated voting game $\Gamma_{x}^{\prime}\left(S,\left(M_{i}\right)_{i \in S}, g\right)$.

(b) There is a truthful equilibrium rejection of $x$ in a (direct) mediated voting game $\Gamma_{x}^{\prime}\left(S,\left(T_{i}\right)_{i \in S}, y\right)$.

(c) There exists $y^{\prime} \in \mathcal{A}_{S}$ and $E \subseteq T$ (with $q(E)>0$ ) such that:

(i) $U_{i}\left(y^{\prime} \mid t_{i}, E\right) \geq U_{i}\left(x \mid t_{i}, E\right)$ for all $i \in S$ and all $t_{i} \in E_{i}$, with strict inequality for some $i$ and $t_{i}$.

(ii) $\sum_{t_{-i} \in E_{-i}\left(t_{i}\right)} q(t)\left[u_{i}\left(y_{i}(t), t\right)-u_{i}\left(x_{i}(t), t\right)\right] \geq$ $\sum_{t_{-i} \in E_{-i}\left(t_{i}^{\prime}\right)} q(t)\left[u_{i}\left(y_{i}\left(t_{-i}, t_{i}^{\prime}\right), t\right)-u_{i}\left(x_{i}(t), t\right)\right]$ for all $i \in S, t_{i}, t_{i}^{\prime} \in T_{i}$.

Again, we can use the equivalence between (a) and (b) to claim that several versions of the coalitional voting game are strategically equivalent. One version, that we will find convenient in terms of fixing ideas is the 
following. All agents in $S$ report their types, and then the mediator asks for a vote if and only if the reported types belong to $E_{S} \subseteq T_{S}$.

Proof of Proposition 3. Suppose $\sigma=\left(v_{i}\left(t_{i}\right), m_{i}\left(t_{i}\right)\right)$ is an equilibrium of $\Gamma_{x}^{\prime}\left(S,\left(M_{i}\right)_{i \in S}, g\right)$. Let $y(t)=\phi(m(t))$ for all $t$. Of course, $v_{i}\left(t_{i}\right)=\{a\}$ for all $t_{i} \in E_{i}(\sigma)$. Moreover, if we modify $\sigma$ to $\sigma^{\prime}$ only by having all types vote $a, \sigma^{\prime}$ remains an equilibrium. It now follows that in the direct mediated mechanism $\Gamma_{x}^{\prime}\left(S,\left(T_{i}\right)_{i \in S}, y\right)$, it is an equilibrium for all types of agents in $S$ to accept, and report truthfully. To see this, note that voting to reject the alternative mechanism yields the status-quo - an option that was feasible in the original mechanism. A unilateral deception also yields an outcome that is feasible in the original mechanism. Clearly then, (a) and (b) are equivalent.

Suppose $\sigma$ is a truthful equilibrium rejection of $x$ in the game $\Gamma_{x}^{\prime}\left(S,\left(T_{i}\right)_{i \in S}, y\right)$. This means that:

- No $i \in S$ of type $t_{i}$ can gain by rejecting the alternative mechanism. For any $t_{i} \notin E_{i}(\sigma)$ the accept/reject decision is outcome equivalent. For $t_{i} \in E_{i}(\sigma)$, we must have $U_{i}\left(y \mid t_{i}, E\right) \geq U_{i}\left(x \mid t_{i}, E\right)$. Thus,

$$
U_{i}\left(y \mid t_{i}, E\right) \geq U_{i}\left(x \mid t_{i}, E\right) \text { for all } i \in S \text { and all } t_{i} \in E_{i}
$$

with strict inequality for some $i$ of some type.

- No $i \in S$ of type $t_{i}$ can gain by pretending to be of type $t_{i}^{\prime}$ (incentive compatibility). The interim utility from truthful reporting is:

$$
U_{i}\left(\sigma \mid t_{i}\right)=\sum_{t_{-i} \in E_{-i}\left(t_{i}\right)} q(t) u_{i}\left(y_{i}(t), t\right)+\sum_{t_{-i} \notin E_{-i}\left(t_{i}\right)} q(t) u_{i}\left(x_{i}(t), t\right) .
$$

If agent $i$ of type $t_{i}$ reports $t_{i}^{\prime}$, the resulting interim utility is: ${ }^{11}$

$$
U_{i}\left(\sigma, t_{i}^{\prime} \mid t_{i}\right)=\sum_{t_{-i} \in E_{-i}\left(t_{i}^{\prime}\right)} q(t) u_{i}\left(y_{i}\left(t_{-i}, t_{i}^{\prime}\right), t\right)+\sum_{t_{-i} \notin E_{-i}\left(t_{i}^{\prime}\right)} q(t) u_{i}\left(x_{i}(t), t\right) .
$$

And we must have

$$
U_{i}\left(\sigma \mid t_{i}\right) \geq U_{i}\left(\sigma, t_{i}^{\prime} \mid t_{i}\right) \text { for all } i \in S \text {, for all } t_{i}, t_{i}^{\prime} \in T_{i} .
$$

\footnotetext{
${ }^{11}$ Note how the different type report affects the implementation of the blocking plan, but not that of the status-quo.
} 
This general incentive compatibility constraint can also be expressed in a form that compares utilities only over the relevant subset of $E(\sigma)$. To do this, subtract $U_{i}\left(x \mid t_{i}\right)$ from the right hand side of both $U_{i}\left(\sigma \mid t_{i}\right)$ and $U_{i}\left(\sigma, t_{i}^{\prime} \mid t_{i}\right)$ to re-state the above inequality as:

$$
\begin{aligned}
& \sum_{t_{-i} \in E_{-i}\left(t_{i}\right)} q(t)\left[u_{i}\left(y_{i}(t), t\right)-u_{i}\left(x_{i}(t), t\right)\right] \geq \\
& \sum_{t_{-i} \in E_{-i}\left(t_{i}^{\prime}\right)} q(t)\left[u_{i}\left(y_{i}\left(t_{-i}, t_{i}^{\prime}\right), t\right)-u_{i}\left(x_{i}(t), t\right)\right] \text { for all } i \in S, t_{i}, t_{i}^{\prime} \in T_{i} .
\end{aligned}
$$

This proves that (b) implies (c). The converse follows easily from the same arguments as above by considering the direct mediated mechanism $y^{\prime}$ where:

$$
y^{\prime}(t)= \begin{cases}y(t) & \text { if } t \in E \\ x(t) & \text { otherwise }\end{cases}
$$

To compare Proposition 3 to Proposition 2, consider $x \in \mathcal{A}^{*}$ and $y, E$ satisfying condition (c) of Proposition 3. For $t_{i}^{\prime} \notin E_{i}, E_{-i}\left(t_{i}^{\prime}\right)=\emptyset$ (or more precisely, consists of types that have 0 probability given $\left.t_{i}\right)$. The RHS of (c.ii) is then 0 , and the condition becomes the same as (c.i). Thus, we need only consider (c.ii) for those cases in which $t_{i}^{\prime} \in E_{i}$.

It may now be useful to write (c.ii) explicitly for the remaining two cases:

(i) $t_{i} \notin E_{i}$ and $t_{i}^{\prime} \in E_{i}$ : in this case, the LHS is 0, and (c.ii) becomes the same as (c.iii) of Proposition 2; the self selection condition used in defining a credible objection.

(ii) $t_{i} \in E_{i}$ and $t_{i}^{\prime} \in E_{i}$ : in this case, if $E$ has a product structure, $E_{-i}\left(t_{i}\right)=E_{-i}\left(t_{i}^{\prime}\right)$ and it is easy to see that (c.ii) of Proposition 3 is the same as (c.ii) of Proposition 2.

Thus, we see that the (c) of Proposition 2 implies (c) of Proposition 3, and the converse holds if $E$ is a product event. This leads us to define the mediated core as the set of all allocations in $\mathcal{A}^{*}$ for which there does not exist a coalition $S$, and event $E$ and an allocation $y \in \mathcal{A}_{S}$ satisfying condition (c) of Proposition 3. Clearly then, the mediated core is a subset of the credible core. Recall the analogy from the introduction: a credible objection is to the independent choice of actions in a Nash equilibrium just like a mediated objection is to the action choice in a correlated equilibrium. The next example illustrates the difference between credible core and mediated core, and shows that the mediated core can be a strict subset of the credible core. 
Example 2: Consider an exchange economy with three agents and three commodities. Agent 3 is uninformed and agents 1 and 2 have two types each: $T_{1}=\left\{t_{1}, t_{1}^{\prime}\right\}, T_{2}=\left\{t_{2}, t_{2}^{\prime}\right\}$ and each state is equally likely. Let $t=\left(t_{1}, t_{2}\right)$ and $t^{\prime}=\left(t_{1}^{\prime}, t_{2}^{\prime}\right)$. The state independent endowments are:

$$
\omega_{1}=(1,0,0), \quad \omega_{2}=(0,1,0), \quad \omega_{3}=(0,0,2) .
$$

The utility functions are:

$$
\begin{aligned}
& u_{1}((x, y, z), t)=0.9 x+y+1.05 z \\
& u_{1}\left((x, y, z), t^{\prime}\right)=5 z \\
& u_{1}\left((x, y, z),\left(t_{1}^{\prime}, t_{2}\right)\right)=u_{1}\left((x, y, z),\left(t_{1}, t_{2}^{\prime}\right)\right)=0.9 x+y \\
& u_{2}((x, y, z), t)=x+0.9 y+1.05 z \\
& u_{2}\left((x, y, z), t^{\prime}\right)=5 z \\
& u_{2}\left((x, y, z),\left(t_{1}^{\prime}, t_{2}\right)\right)=u_{2}\left((x, y, z),\left(t_{1}, t_{2}^{\prime}\right)\right)=x+0.9 y \\
& \quad u_{3}((x, y, z), s)=x+y \text { for all } s .
\end{aligned}
$$

The status-quo allocation $x$, along with ex-post utilities, is the following:

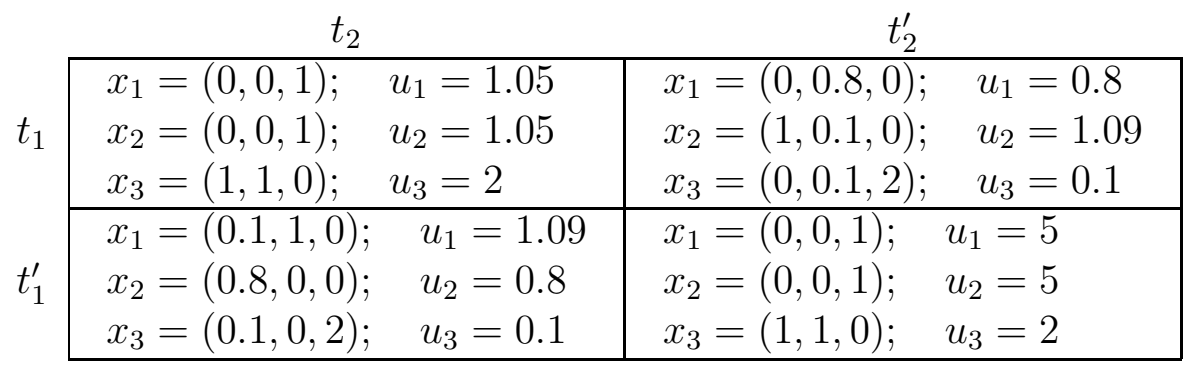

This allocation rule is incentive compatible, individually rational and interim efficient. Furthermore, coalition $\{1,2\}$ does not have a coarse objection because type $t_{1}^{\prime}$ and $t_{2}^{\prime}$ cannot be better-off without commodity $z$. Also, it is easy to see that coalitions $\{1,3\}$ or $\{2,3\}$ do not have a coarse objection: there are not enough units of goods 1 or 2 to improve upon player 3's expected utility. This shows that $x$ is in the incentive compatible coarse core.

Moreover, there is no credible objection to $x$ either. To see this suppose there is a credible objection by agents 1 and 2 over some event $E$. If the event is $t$ or $t^{\prime}$ they cannot do better. If the event is $\left(t_{1}^{\prime}, t_{2}\right)$ they can do better, but then type $t_{1}$ will want to lie; the preferences of agent 1 are the same in $\left(t_{1}, t_{2}\right)$ and $\left(t_{1}^{\prime}, t_{2}\right)$. The event $E$ cannot be the first column either, because agent 1 will have to be given utility at least 1.09 in each state (to 
make sure that she is better-off in state $\left(t_{1}^{\prime}, t_{2}\right)$, and to maintain incentive compatibility). But then $t_{2}$ would be worse off, getting no more than a utility of 0.9 in each state. For similar reasons, the argument holds for state $\left(t_{1}, t_{2}^{\prime}\right)$ or the first row. This exhausts all possibilities for a credible objection. Thus $x$ belongs to the credible core.

Now consider a mediated objection in which the mediator will use the mechanism $\delta$ for coalition $\{1,2\}$ in states other than $t^{\prime}$ :

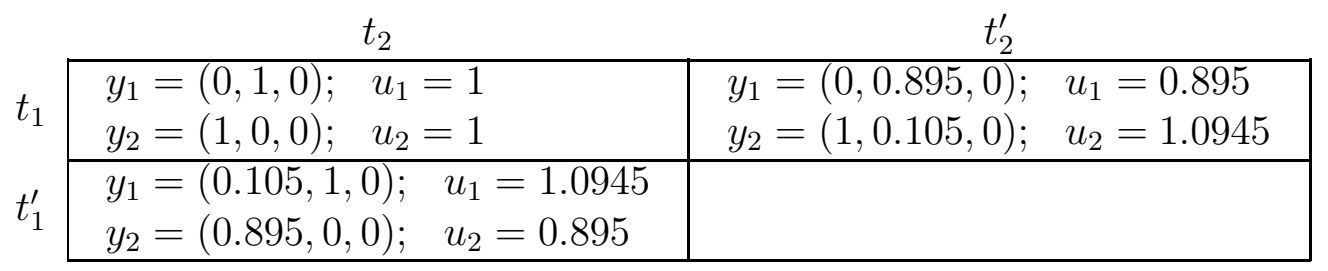

Type $t_{1}$ 's expected utility from the blocking plan is higher than at the status-quo: he gets a utility equal to 1 in state $\left(t_{1}, t_{2}\right)$ and equal to 0.895 in state $\left(t_{1}, t_{2}^{\prime}\right)$ (instead of status-quo utilities 1.05 and 0.8 , respectively): since he does not learn anything from the call of the mediator, he maintains the same beliefs to sustain a higher expected utility in the blocking plan. On the other hand, type $t_{1}^{\prime}$ learns from the call of the mediator that the state is $\left(t_{1}^{\prime}, t_{2}\right)$, and in that state the blocking plan utility is 1.0945 , higher than the status-quo utility of 1.09. The arguments for types $t_{2}$ and $t_{2}^{\prime}$ are identical to those for $t_{1}$ and $t_{1}^{\prime}$, respectively.

It only remains to check for incentive compatibility of the blocking plan. Let us begin with type $t_{1}$. Again, by being truthful, his expected utility will be $(1 / 2)(1+0.895)$. If he misreports his type by announcing $t_{1}^{\prime}$ to the mediator, he will be invited to the blocking plan only with probability $1 / 2$ when the type of player 2 is $t_{2}$ and he will then be offered to consume $(0.105,1,0)$, whereas with the rest of probability he will not get the phone call and the status-quo will result. However, in this case, his false report will not interfere with the status-quo since, to implement it, the very first (truthful) reports are used. In conclusion, in this case he is allocated $(0,0.8,0)$. The corresponding expected utility is $(1 / 2)(1.0945+0.8)$, smaller than what he gets by being truthful. In other words, type $t_{1}$ does not gain by lying, because the gain in the first column is 0.0945 , but the loss in the second column is 0.095. Finally, let us check the incentives of type $t_{1}^{\prime}$. If he reports truthfully, with probability $1 / 2$ he will be invited to the objection and be allocated $(0.105,1,0)$ [in state $\left(t_{1}^{\prime}, t_{2}\right)$ ], while with probability $1 / 2$ he will be allocated 
$(0,0,1)$ [in state $\left(t_{1}^{\prime}, t_{2}^{\prime}\right)$ from the status-quo, where he really values good $z$ ]. This is better than being allocated $(0,1,0)$ and $(0,0.895,0)$, respectively. Of course, the incentive compatibility arguments for types $t_{2}$ and $t_{2}^{\prime}$ are identical to the corresponding types of player 1 , and we omit them.

Since the blocking inequalities we have written are all strict, we therefore conclude that $x$ is not in the (weak) mediated core, and therefore not in the mediated core either.

\section{Randomized Mediation}

In this section we consider coalitional voting in which the mechanism may involve an even more sophisticated role for the mediator. A mediator may construct a blocking plan in which the coalition that is asked to vote on a new alternative mechanism is chosen at random. This corresponds closely to a core concept recently suggested by Myerson (2003). ${ }^{12}$

Given Propositions 2 and 3, it should be clear that without loss of generality we may concentrate on direct voting games in which agents vote and report their types. A particular version of such games that we will find convenient to refer to is the one in which agents first report their types, and are then asked for a vote (as a function of the type reports).

Now, a proposal by a mediator, $\mu$, consists of a probability distribution of feasible allocation mechanisms for various coalitions. In particular, $\mu\left(S, y^{S}, t\right)$, where $y^{S} \in \mathcal{A}_{S}$, denotes the probability with which coalition $S$ is invited by the mediator to vote for $y$ against the status-quo, when the (reported) state is $t$. Recall that, for ease of comparisons with the previous sections we will consider randomization only over coalitions, i.e., $\mu\left(S, y^{S}, t\right)>0$ implies $\mu\left(S, z^{S}, t\right)=0$ for all $z^{S} \neq y^{S}$. Thus, we associate with each coalition $S$ one proposed allocation $y^{S} \in \mathcal{A}_{S}$. The mediator may choose with positive probability not to invite any coalition, thereby imposing the statusquo. Thus, for each $t \in T, 0 \leq \mu\left(S, y^{S}, t\right) \leq 1$ for all coalitions $S$, and $\sum_{S} \mu\left(S, y^{S}, t\right) \leq 1$. This describes a 'blocking plan' used in the definition and characterization of the inner core; see Myerson (1991), Qin (1989) and de Clippel and Minelli (2002).

\footnotetext{
${ }^{12}$ To maintain comparability with the previous sections we neglect the possibility that the alternative mechanism may be a random state contingent allocation. Myerson (2003) allows for this form of randomization as well.
} 
To apply this idea to coalitional voting with incomplete information, we consider the following extension of the game $\Gamma_{x}^{\prime}$ of the previous section. Given a status-quo $x$ and a blocking plan $\mu$, all agents vote whether to accept or reject $\mu$ and report their types to the mediator, who then forms coalitions according to the plan $\mu$ : given the reports, say $t$, the mediator chooses coalition $S$ with probability $\mu\left(S, y^{S}, t\right)$. If all voters accept, the new proposal is implemented according to the plan $\mu$, while otherwise the status-quo survives. Note that at the time of taking the vote each agent must use $\mu$ to update his beliefs at his information set: in particular, he must take into account that he has received the call from the mediator, something that, according to $\mu$, need not happen with probability 1 . As with the games $\Gamma_{x}^{\prime}$, we assume that the voting game is played only by those players that have been asked to participate in the blocking move, after they have been asked.

Based on this interpretation of a mediator, we are led to modifying the notion of mediated core of the previous section as follows.

An allocation $x \in \mathcal{A}^{*}$ is said to belong to the randomized mediated core if there does not exist a blocking plan $\mu$ such that

$\sum_{t_{-i}} q(t) \sum_{S \supseteq\{i\}} \mu\left(S, y^{S}, t\right)\left[u_{i}\left(y_{i}^{S}(t), t\right)-u_{i}\left(x_{i}(t), t\right)\right] \geq 0$ for all $i \in N$ and $t_{i} \in T_{i}$

and

$\sum_{t_{-i}} q(t) \sum_{S \supseteq\{i\}} \mu\left(S, y^{S}, t\right)\left[u_{i}\left(y_{i}^{S}(t), t\right)-u_{i}\left(x_{i}(t), t\right)\right] \geq$

$\sum_{t_{-i}} q(t) \sum_{S \supseteq\{i\}} \mu\left(S, y^{S}, t_{-i}, t_{i}^{\prime}\right)\left[u_{i}\left(y_{i}^{S}\left(t_{-i}, t_{i}^{\prime}\right), t\right)-u_{i}\left(x_{i}(t), t\right)\right]$ for all $i \in N$ and $t_{i}, t_{i}^{\prime} \in T_{i}$.

Suppose $\mu$ is a blocking plan that involves one coalition $S$ and one alternative, $y$, i.e., $\mu\left(S^{\prime}, y^{\prime}\right)=0$ if $S^{\prime} \neq S$ or $y^{\prime} \neq y$. Let $E(\mu)=\{t \in T \mid$ $y(t) \neq x(t)\}$. Then it follows that $\mu$ satisfies the above inequalities if and only if $(S, y, E)$ satisfies condition (c) of Proposition 3. Thus equilibria of deterministic blocking plans are precisely the equilibria of voting games $\Gamma_{x}^{\prime}$ defined in the previous section. Hence, we have:

Observation: The randomized mediated core is a subset of the mediated core, itself a subset of the credible core.

As in the previous section, we can consider resilience to randomized mediated voting by considering random alternative mechanisms $\mu$ to get an alternative characterization of the randomized mediated core. We will omit the corresponding statement. 
The concept of randomized mediated core of the present section is very similar to the core concept defined by Myerson (2003). The only differences are that: (1) he also allows for random allocation rules within each coalition, and (2) he assumes that transfers are possible and feasibility is weakened to require expected feasibility of the transferable commodity. Clearly, in terms of a definition of blocking feature (1) may be significant, as the feasible set for coalitions is the set of lotteries over allocations, while feature (2) is a technical assumption on the environments that Myerson (2003) uses to prove existence.

We close the section by going over another example, to illustrate the difference between the randomized mediated core and other cores previously mentioned.

Example 3: This example is an adaptation of Myerson's (2003) Example 1 to our framework. The set of agents is $N=\{1,2\}$, and agent 2 is uninformed. Let $T_{1}=\left\{t_{H}, t_{L}\right\}$ be the set of types of agent 1 . Both types are equally likely. Agent 1 is a seller of good $x$, to be exchanged for money (good $y$ ). The state-independent endowment is

$$
\omega_{1}=(1,0), \quad \omega_{2}=(0,10) .
$$

The utility functions are as follows:

$$
\begin{gathered}
u_{1}\left((x, y), t_{H}\right)=5 x+y, \\
u_{1}\left((x, y), t_{L}\right)=x+y ; \\
u_{2}\left((x, y), t_{H}\right)=6 x+y-10, \\
u_{2}\left((x, y), t_{L}\right)=2 x+y-10 .
\end{gathered}
$$

Consider the following allocation:

$x_{1}\left(t_{H}\right)=(0.75,1.3), \quad x_{2}\left(t_{H}\right)=(0.25,8.7) x_{1}\left(t_{L}\right)=(0,2.05), \quad x_{2}\left(t_{L}\right)=(1,7.95)$.

This allocation is interim incentive efficient and interim individually rational. Therefore, it is in the incentive compatible coarse core. Furthermore, because agent 1 is fully informed, it is easy to see that any allocation in the incentive compatible coarse core is also in the credible core. Since in this economy all events over which objections can be constructed have a product structure, there are no additional mediated objections. Hence, the mediated core also coincides with the credible core. Therefore, $x$ is in the mediated core of the previous section. 
Let us see now that $x$ is not in the randomized mediated core. Consider the following blocking plan $\mu$ : $\mu\left(\{1,2\}, y^{\{1,2\}}, t_{H}\right)=0.25, \mu\left(\{1\}, y^{\{1\}}, t_{H}\right)=$ 0.75 , while $\mu\left(S, y^{S}, t_{L}\right)=0$ for all $S$, where

$$
y_{1}^{\{1,2\}}\left(t_{H}\right)=(0,5.2), \quad y_{2}^{\{1,2\}}\left(t_{H}\right)=(1,4.8) y_{1}^{\{1\}}\left(t_{H}\right)=(1,0) .
$$

In words, the blocking plan only concerns state $t_{H}$. In it, coalition $\{1,2\}$ will be formed with probability 0.25 and exchange the entire unit of good $x$ for 5.2 units of money, while with probability 0.75 agent 1 will be instructed to keep his initial endowment.

In the corresponding voting game, the following profile is a Bayesian Nash equilibrium: let each agent accept the blocking plan and report his type truthfully. Let us check this:

- Agent 1 of type $t_{L}$ is being excluded from the blocking plan $\mu$. Therefore, the expected utility that he receives from playing his equilibrium strategy is the status-quo utility 2.05. Changing his vote to a rejection would not alter this outcome. Finally, misreporting his type and pretending to be type $t_{H}$ would yield an expected utility $0.25 \cdot 5.2+0.75 \cdot 1=2.05$, so he is at a best response by telling the truth.

- Agent 1 of type $t_{H}$ joins the blocking plan in this equilibrium, and his expected utility from doing so is $0.25 \cdot 5.2+0.75 \cdot 5=5.05$, which is also his status-quo expected utility. Thus, he would not benefit from rejecting the blocking plan. Misreporting his type would also result in the status-quo, and thus, he is also at a best response.

- Agent 2's expected utility from accepting the blocking plan is simply $6-5.2$, i.e., he learns from having to vote that the state is $t_{H}$ and that coalition $\{1,2\}$ has been called to exchange one unit of $x$ for 5.2 units of money. Therefore, if he accepts, he ends up with the bundle $(1,4.8)$. which is better than what he would get from the status quo by rejecting the blocking plan (the bundle $(0.25,8.7)$ ).

Note finally how in this example the weak inequalities in the objection make a big difference. That is, while we have shown that $x$ is not in the randomized mediated core, we claim that it is in its weak version defined by objections that use strict inequalities for every type. To see this, note that 
because of incentive compatibility, if one improves type $t_{H}$ strictly, type $t_{L}$ will also want to join. This means that he needs to be part of the blocking plan and also strictly improve, which renders improving the buyer impossible.

\section{Relationship to Durability}

We close the paper by relating our approach to durability. In their analysis of interim efficiency, Holmström and Myerson (1983) point out that agents may sometimes discard an interim (incentive) efficient allocation in favor of another allocation. ${ }^{13}$ Their notion of durability is meant to formalize the following:

"The essential idea .... is that an incentive compatible decision rule $\delta$ should be considered durable iff the individuals in the economy would never unanimously approve a change from $\delta$ to any other decision rule." (Holmström and Myerson (1983, p. 1811))

Their actual definition of durability proceeds as follows. Given an allocation $x$ and an alternative feasible allocation $y$, they consider a voting game $\Gamma_{x}\left(N,\left(T_{i}\right)_{i \in N}, y\right)$ as in our Subsection 3.2, in which type reports are made after it is determined whether or not $y$ is unanimously accepted. An allocation $x$ is said to endure $y$ if there exists an equilibrium in which $y$ is rejected in every state. Since there always exists a Bayesian equilibrium in which everyone rejects the alternative because they do not expect to be pivotal, it becomes necessary to refine the equilibrium notion. They do this by appealing to some of the conditions used in defining a trembling hand perfect equilibrium. An allocation $x \in \mathcal{A}^{*}$ is said to be durable if it endures every alternative mechanism in $\mathcal{A}$. Thus, a durable allocation has the property that for every alternative, there exists an equilibrium rejection of that alternative.

To compare our approach with durability, we apply the stability notion implicit in $\Gamma_{x}$ of Subsection 3.2 to the grand coalition. An allocation $x \in \mathcal{A}^{*}$ is said to be resilient to grand coalition voting, or simply resilient if there is no equilibrium rejection of $x$ in a voting game $\Gamma_{x}\left(N,\left(T_{i}\right)_{i \in N}, y\right)$.

Using the proof of Proposition 2 applied to $S=N$, it is easy to see that every resilient allocation is interim incentive efficient. In general, the other

\footnotetext{
${ }^{13}$ This phenomenon should be seen as an important consequence of incomplete information since it is, of course, impossible in the complete information setting.
} 
implication does not hold; to see this, note that one can find an event $E$, proper subset of $T$, over which the relevant types enjoy an improvement. It is true, though, that a uniformly incentive compatible interim incentive efficient allocation is resilient (see Dutta and Vohra (2003)).

The reader will notice that our notion of resilience seems to be more faithful to the idea described by the above Holmstróm-Myerson quote. A resilient allocation has the property that there is no equilibrium in which the alternative is ever unanimously accepted in place of the given status-quo. To meet the durability test it is enough that there exist a (trembling-hand perfect) equilibrium such that in every positive probability state the alternative is rejected. It does not rule out the possibility that there is also an equilibrium in which the alternative is accepted. Clearly, if there exists no equilibrium (perfect or not) in which the alternative is accepted with positive probability, then provided an equilibrium exists, it must involve a rejection of the alternative in each (positive probability) state. In this sense, a resilient allocation is durable. But the converse need not be true. This can be illustrated by the example in section 9 of Holmström and Myerson (1983) in which an inefficient allocation is durable because there is an equilibrium in which the status-quo endures the alternative. But there also exists, for an alternative that is an interim improvement, an equilibrium in which the alternative is unanimously accepted. Of course, this implies that the original allocation was not resilient. The basic idea in our approach, given our interest in a cooperative solution such as the core, is that a coalition may choose any feasible allocation as long as it can be supported by an equilibrium. In particular, a coalition should be able to resolve any coordination problem (potentially found in situations like those in Holmström and Myerson's (1983) example of Section 9). Compared to durability, this makes it easier to challenge a status-quo, and yields therefore a smaller set of stable allocations. Not surprisingly, this may lead to problems of non-existence. But in studying the core, unlike efficient allocations, this is a problem that we cannot avoid in general, anyway (see Vohra(1999)). 


\section{References}

Cramton, P. and T. Palfrey (1995), "Ratifiable Mechanisms: Learning from Disagreement", Games and Economic Behavior, 10, 255-283.

de Clippel, G. (2004), "The Type-Agent Core of Exchange Economies with Asymmetric Information," Mimeo, Department of Economics, Brown University.

de Clippel, G. and E. Minelli (2002), "Two Remarks on the Inner Core", CORE discussion paper 2002/01.

Dutta, B. and R. Vohra (2003), "Incomplete Information, Credibility and the Core." Working Paper No. 2003-21, Department of Economics, Brown University.

Forges, F., E. Minelli and R. Vohra (2002), "Incentives and the Core of an Exchange Economy: A Survey." Journal of Mathematical Economics, 38, $1-41$.

Forges, F., J.-F. Mertens and R. Vohra (2002), "The Ex Ante Incentive Compatible Core in the Absence of Wealth Effects", Econometrica 70, 1865-1892.

Holmström, B. and R. Myerson (1983), "Efficient and Durable Decision Rules with Incomplete Information." Econometrica 51, 1799-1819.

Lee, D. and O. Volij (2002). "The Core of Economies with Asymmetric Information: An Axiomatic Approach." Journal of Mathematical Economics, $38,43-63$.

Maskin, E. and J. Tirole (1992), "The Principal-Agent Relationship with an Informed Principal. Part 2: Common Values," Econometrica 60, 1-42.

Myerson, R. (1991), Game Theory: Analysis of Conflict Harvard University Press.

Myerson, R. (2003), "Virtual Utility and the Core for Games with Incomplete Information." Mimeo, University of Chicago.

Perry, M. and P. Reny 1994), "A Non-Cooperative View of Coalition Formation and the Core," Econometrica, 62, 795-817.

Qin, C-Z. (1993), "The Inner Core and the Strictly Inhibitive Set", Journal of Economic Theory, 59, 431-444.

Serrano, R. and R. Vohra (1997), "Non-Cooperative Implementation of the Core." Social Choice and Welfare, 14, 513-525.

Vohra, R. (1999), "Incomplete Information, Incentive Compatibility and the Core." Journal of Economic Theory 86, 123-147. 
Volij, O. (2000), "Communication, Credible Improvements and the Core of an Economy with Asymmetric Information." International Journal of Game Theory 29, 63-79.

Wilson, R. (1978), "Information, Efficiency and the Core of an Economy." Econometrica 46, 807-816. 